\title{
Magnolia dealbata en Nuevo León, México
}

\section{Magnolia dealbata in Nuevo León, México}

\author{
Carlos Gerardo Velazco-Macías ${ }^{1 *}$, Rahim Foroughbakhch-Pournavab², Glafiro José Alanís-Flores² y Marco \\ Antonio Alvarado-Vázquez ${ }^{2}$ \\ ${ }^{1}$ Av. Eugenio Garza Sada 6604, Col. Lagos del Bosque, 64890 Monterrey, Nuevo León, México. \\ ${ }_{2}^{2}$ Facultad de Ciencias Biológicas, Universidad Autónoma de Nuevo León, 66451 San Nicolás de los Garza, Nuevo León, México. \\ *Correspondencia: carlos.velazco@gmail.com
}

\begin{abstract}
Resumen. Se analiza y describe la distribución de Magnolia dealbata Zucc. en el estado de Nuevo León, México, aportando información acerca de la ecología, estado de conservación, situación del hábitat y fenología de las poblaciones estudiadas. Por medio de una revisión bibliográfica y trabajo de campo realizado en la sierra Madre Oriental, se obtuvieron datos que permiten distinguir 2 núcleos poblacionales ubicados en el municipio de Montemorelos, en el centro del estado, con extensión total de 17.66 hectáreas, y densidad de hasta 30 individuos por $100 \mathrm{~m}^{2}$. Se presenta en asociación con Pinus teocote, Quercus spp., Cornus florida, Carya sp. y Sambucus nigra. La observación fenológica durante el periodo de estudio mostró que la aparición de nuevos brotes foliares comienza a principios de marzo y la floración a principios de mayo. El hábitat se observa con pocas alteraciones y no se identificaron amenazas a corto plazo para estas poblaciones.
\end{abstract}

Palabras clave: Magnolia, México, ecología, conservación.

\begin{abstract}
The distribution of Magnolia dealbata Zucc. in the state of Nuevo León in northeastern Mexico is analyzed and described; information regarding its conservation status, habitat health, and phenology of studied populations is provided. Through bibliographical information and field work in the Sierra Madre Oriental, 2 core populations of M. dealbata Zucc. can be distinguished, both located in the municipality of Montemorelos in the center of the state, covering a total of 17.66 hectares. Density reaches 30 individuals per 100 square meters. The species grows in association with Pinus teocote, Quercus spp, Cornus florida, Carya sp. and Sambucus nigra. New leaves sprout in early March, and flower production starts in early May. Habitat shows little disturbance and no short-term threats were identified for these populations.
\end{abstract}

Key words: Magnolia, Mexico, ecology, conservation.

\section{Introducción}

Magnolia dealbata Zucc. es un elemento propio de los bosques de niebla y endémico de México, cuyos individuos en la naturaleza se restringen solamente a 6 poblaciones, en estados del centro y este de México (Oaxaca, Querétaro, Veracruz, Hidalgo y San Luis Potosí), las cuales se componen de unos cuantos individuos, con excepción de la población de Coyopola en Veracruz (Luna, 2003; Sánchez-Velázquez y Pineda-López, 2006). Esta especie es el único representante de la sección Rhytidospermum del género en México, y su carácter único ha sido confirmado por estudios moleculares que la separan de manera concreta del resto de las especies nativas de México (Azuma et al., 2001; Kim et al., 2001). Por medio de las exploraciones y del trabajo botánico de investigadores en Nuevo León,

Recibido: 24 enero 2007; aceptado: 14 febrero 2008 específicamente en la sierra Madre Oriental, se logró ubicar poblaciones de elementos relictos del bosque de niebla en los municipios de Santiago, Allende y Montemorelos; entre estos elementos destaca Magnolia dealbata (ValdezTámez et al, 2003). El hallazgo es de especial relevancia para la conservación de esta especie (Fig. 1), por lo que se plantea la necesidad de conocer la distribución, ubicación y extensión de la población, así como de llevar a cabo un estudio preliminar sobre la estructura de la población, estado del hábitat, especies asociadas y riesgos para la especie.

Dadas las condiciones restringidas de las poblaciones naturales y el bajo número de individuos presentes en ellas, esta especie está situada en diversas categorías de riesgo en la clasificación nacional e internacional (Vovides, 1998). Actualmente, la legislación mexicana la considera en peligro de extinción en la NOM-059-SEMARNAT-2001 (DOF, 2002) y se encuentra en la categoría EN del IUCN Red Data Book (1994). 


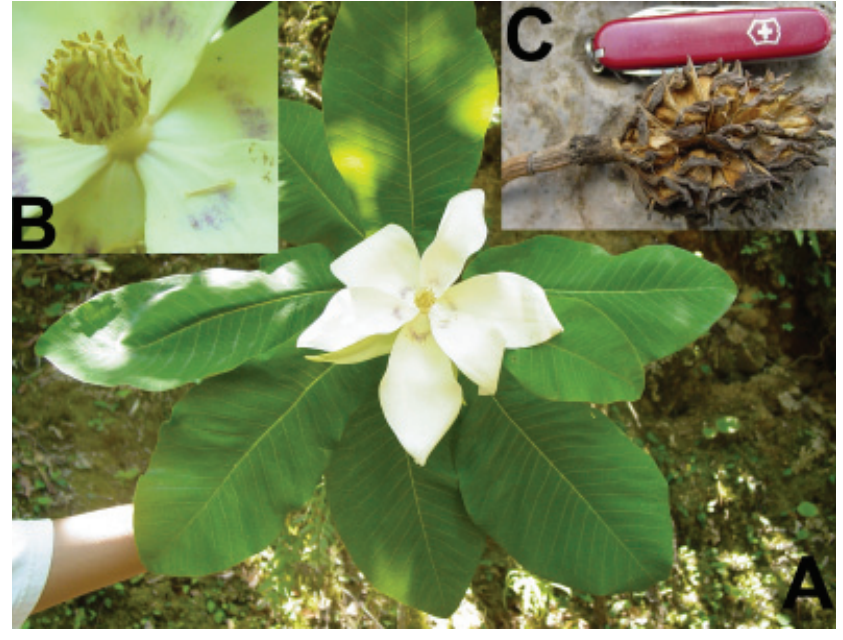

Figura 1. A, ejemplar silvestre de Magnolia dealbata Zucc. en Nuevo León; B, detalle de la flor; C, fruto.

Magnolia dealbata ha sido estudiada taxonómicamente (Vázquez, 1990; 1994), mientras que los aspectos de ecología, distribución, conservación y estado del hábitat han sido tratados de manera específica por Gutiérrez y Vovides (1994), Ramírez et al. (2005), y Corral y Sánchez (2006).

\section{Materiales y métodos}

El área de estudio se ubica en el centro oeste del estado de Nuevo León, en el municipio de Montemorelos, muy cerca de los límites con el estado de Coahuila de Zaragoza; las poblaciones de Magnolia dealbata se localizan en la provincia de la sierra Madre Oriental, la cual se caracteriza por tener un clima de tipo $(\mathrm{A}) \mathrm{C}\left(\mathrm{w}_{1}\right)$, templado, semicaálido, subhúmedo, con lluvias en verano, con temperatura media anual mayor a $18^{\circ} \mathrm{C}$; la temperatura del mes más frío oscila entre los -3 y $18^{\circ} \mathrm{C}$ y la precipitación del mes más seco es menor a $40 \mathrm{~mm}$ (Secretaría de Programación y Presupuesto, 1981). La geología del área está caracterizada por asociaciones de lutita-arenisca, y caliza-lutita, datadas del Jurásico superior y Cretácico superior respectivamente; en éstas se presentan fracturamiento intenso, intemperismo somero y permeabilidad baja (Secretaría de Programación y Presupuesto, 1978). Los tipos de suelos presentes son regosol calcárico + litosol + luvisol crómico, con una clase textural fina en los $30 \mathrm{~cm}$ superficiales y una fase física lítica; se encuentran también suelos de tipo litosol + rendzina con una clase textural media en los $30 \mathrm{~cm}$ superficiales; la hidrología superficial está caracterizada por manantiales naturales y corrientes intermitentes de agua (Secretaría de Programación y Presupuesto, 1977). La vegetación registrada para el área de estudio es de bosque de pino-encino y matorral subinerme (Secretaría de Programación y Presupuesto, 1977).

A finales del año 2005 y durante el 2006 se realizaron visitas a la localidad donde se encuentran las poblaciones de Magnolia dealbata en Nuevo León con la finalidad de obtener datos referentes al estado del hábitat, fenología, amenazas y una evaluación preliminar de las poblaciones.

Se georreferenciaron las poblaciones existentes para delimitar y obtener el área de distribución precisa. Se marcaron sitios donde se observó una dominancia de Magnolia dealbata por sobre otras especies arbóreas y en estos sitios se trazó un total de 10 cuadrantes de 10 x $10 \mathrm{~m}$ para los conteos de individuos; estos sitios se denominaron áreas de mayor densidad. Las áreas con individuos dispersos, donde Magnolia dealbata solamente se presenta intercalada de manera esporádica entre el resto de los elementos arbóreos fueron determinadas mediante observación directa; estos sitios se denominaron "áreas de menor densidad" Dentro de los cuadrantes se anotaron las especies de flora acompañantes, y el estado fenológico de los ejemplares de Magnolia dealbata.

Durante el trabajo de campo se realizó una búsqueda extensiva de factores que afecten la viabilidad de las poblaciones, así como de los usos tradicionales que le dan los habitantes de comunidades cercanas.

Se utilizaron herramientas de información geográfica para realizar un análisis espacial y así determinar las áreas ocupadas por las poblaciones de Magnolia dealbata a partir de modelos digitales de elevación, capas de datos vectoriales y ortofotos digitales del año de 1995 a una escala de 1:20,000.

La confirmación en la determinación de la especie y su separación de Magnolia macrophylla se realizó utilizando las descripciones y caracteres morfológicos descritos en Hernández-Cerda, 1980; Vázquez, 1990; y Meyer, 1997.

\section{Resultados}

Las poblaciones se localizan en la vertiente este de la sierra Madre Oriental entre la sierras Los Nogales y la de la Cebolla, al sureste del ejido La Trinidad en el municipio de Montemorelos (Fig. 2). Están localizadas entre los 1500 y los 1700 m snm; se encontró que los núcleos densos de individuos se restringen a cañadas profundas y protegidas (Figs. 3 y 4), en las cuales se han contado 30 individuos por $100 \mathrm{~m}^{2}$ como máximo, mientras que el total de individuos contabilizados fue de 81 en $1000 \mathrm{~m}^{2}$ en 2 áreas separadas (áreas de mayor densidad). Si existe un individuo cada 13 


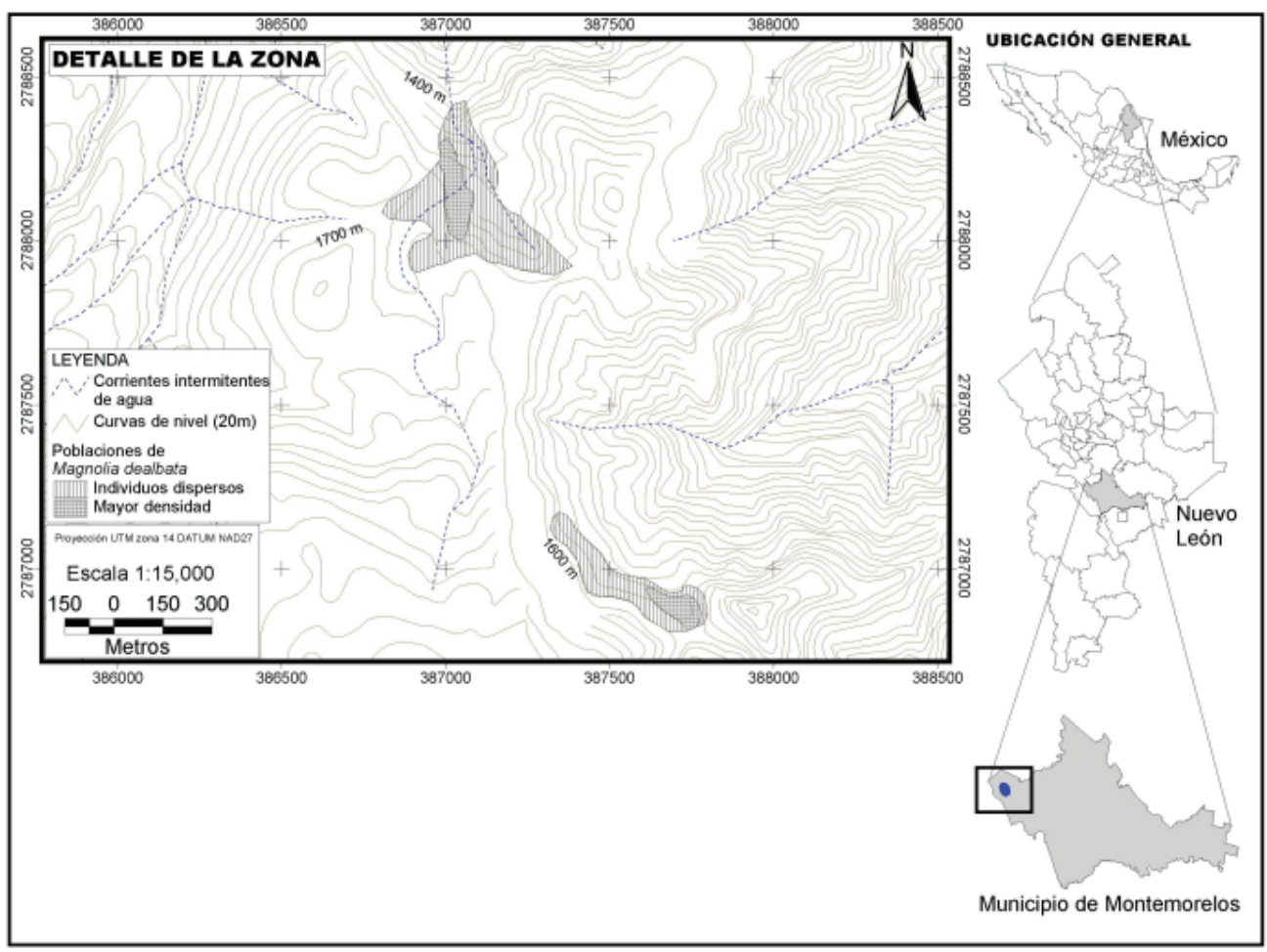

Figura 2. Ubicación geográfica y espacial de las poblaciones de $M$. dealbata en Nuevo León.

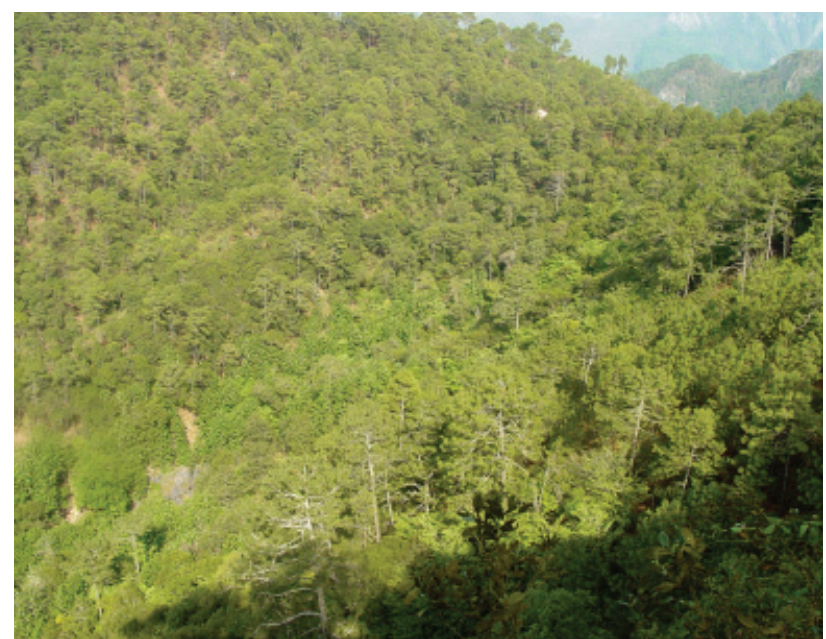

Figura 3. Vista del área 1, con una extensión de 12.53 hectáreas.

$\mathrm{m}^{2}$, las estimaciones más conservadoras muestran que al extrapolar los datos de los 2 núcleos poblacionales, pueden llegar a encontrarse más de 1000 individuos.

Los núcleos poblacionales de Magnolia están inmersos en bosque de Pinus-Quercus o rodeados por él, donde las especies dominantes son Pinus teocote, Quercus spp., Cornus florida y Carya sp.

El área comprobada que está ocupada por las

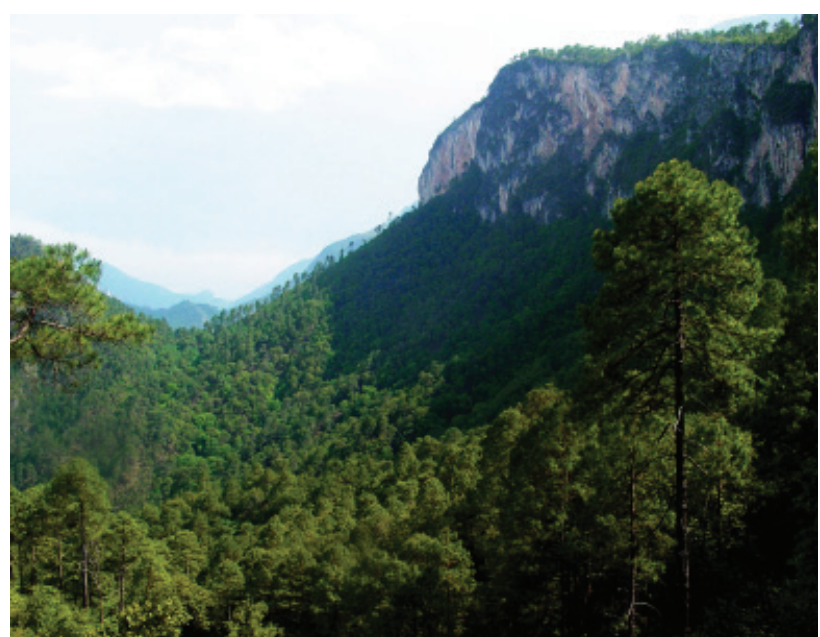

Figura 4. Vista del área 2, con una extensión de 5.13 hectáreas.

poblaciones abarca un total de 17.66 hectáreas, divididas en 2 áreas de forma irregular (Fig. 2), cada una de las cuales cuenta con una zona donde se concentran núcleos densos de individuos y un área con individuos dispersos entre los diversos elementos de flora arbórea (Cuadro 1).

Se encontró que las poblaciones están compuestas por individuos de todas las edades; se observa regeneración por semilla en toda el área, principalmente en sitios donde 
se ha eliminado la cubierta vegetal. La mayoría de los individuos son ejemplares maduros, llegando a encontrarse árboles de hasta $20 \mathrm{~m}$ de altura.

Respecto a la fenología de $M$. dealbata en Nuevo León, durante el periodo de estudio se observó que la producción de hojas se inició a principios de marzo, seguida por la producción de botones florales y flores a principios de mayo. La maduración de los frutos se observó en el periodo comprendido entre finales de junio y principios de julio. Se pudo apreciar también que los individuos de esta especie perdieron las hojas durante los meses de diciembre, enero y febrero.

No se encontró que esta especie sea aprovechada en alguna forma por los pobladores de los ejidos locales, aunque se observan individuos con evidencia de que se ha cortado el tronco principal. No se identificaron amenazas a corto o mediano plazo como plagas, tala clandestina o aprovechamiento tradicional; solamente los incendios forestales permanecen como única amenaza permanente para esta especie y el bosque en el que habita. Cabe destacar que las poblaciones estudiadas están localizadas dentro del Parque Nacional Cumbres de Monterrey, lo cual podría representar una ventaja adicional en la conservación de dicha especie.

Ejemplares examinados: MÉXICO. Nuevo León. Municipio de Montemorelos, ejido La Trinidad, 2511'45',-10006'59', 1600 m snm, 21 marzo 2006, Velazco-Macías, C.G., s.n. (UNL); Ejido La Trinidad, 2512'23' -10007'15', $1500 \mathrm{~m}$ snm, 14 mayo 2006, Velazco-Macías, C.G., s.n. (UNL).

\section{Discusión}

A pesar de que Magnolia dealbata está registrada de Nuevo León (Valdez-Támez et al., 2003), no cuenta con el respaldo de ejemplares de herbario con los que se pueda corroborar la presencia de la especie, esta situación se subsana con parte del trabajo de campo realizado; el hallazgo de una población Magnolia dealbata en el estado de Nuevo León representa un logro en la conservación a largo plazo de esta especie dado que las poblaciones conocidas solamente estaban representadas por unos cuantos individuos, de poco más de 200 ejemplares la más grande, en el estado de Veracruz (Gutiérrez y Vovides, 1997). La población localizada en la sierra Madre Oriental, en el municipio de Montemorelos, está compuesta por 2 núcleos principales de individuos, donde se han contabilizado hasta 30 por $100 \mathrm{~m}^{2}$. Si bien el efecto de microhábitat se ha registrado como un factor importante para la presencia de esta especie (Gutiérrez y Vovides, 1997), para el caso de las poblaciones estudiadas en Nuevo
León, el microhábitat solo se registra en las cañadas donde se encuentran altas densidades de individuos, mientras que en áreas abiertas la especie parece estar bien adaptada a la competencia con otras especies del bosque de pino encino. Estudios previos no hacen mención de los factores físicos o abióticos relevantes para la presencia de esta especie; en Nuevo León, la combinación de factores geológicos y edáficos parecen restringir en cierta medida esta especie, ya que las combinaciones de lutita-arenisca y caliza-lutita, y las mezclas regosol calcárico + litosol + luvisol crómico son los elementos dominantes en el área donde se localizan las poblaciones de Magnolia dealbata y siguen un patrón similar en la distribución.

\section{Agradecimientos}

A los biólogos Liliana Ramírez Freire y Juan Carlos Larumbe Moreno por su colaboración en la realización del trabajo de campo, así como al Ing. Jorge Martínez Pineda por su apoyo en el Sistema de Información Geográfico.

\section{Literatura citada}

Azuma, H., J. G. García-Franco, V. Rico-Gray y L. B. Thien. 2001. Molecular phylogeny of the Magnoliaceae: the biogeography of tropical and temperate disjunctions. American Journal of Botany 88:2275-2285.

Corral-Aguirre, J. y L. R. Sánchez-Velásquez. 2006. Seed ecology and germination treatments in Magnolia dealbata: an endangered species. Flora-Morphology, Distribution, Functional Ecology of Plants 201:227-232.

DOF (Diario Oficial de la Federación). 2002. Norma Oficial Mexicana NOM-059-ECOL-2001, Protección AmbientalEspecies nativas de México de flora y fauna silvestresCategorías de riesgo y especificaciones para su inclusión, exclusión o cambio - Lista de especies en riesgo. 06 marzo, 2002.

Gutiérrez, L. y A. Vovides. 1994. An in situ study of Magnolia dealbata Zucc. in Veracruz State: an endangered endemic tree of México. Biodiversity and Conservation 6:89-97.

Hernández Cerda, M. A. 1980. Magnoliaceae. In Flora de Veracruz, fascículo 14. Instituto Nacional de Investigaciones sobre Recursos Bióticos, Xalapa, Veracruz. p. 1-10.

IUCN (International Union for Conservation of Nature). 1994. Categorías de las listas rojas de la UICN. IUCN, Gland.

Kim, S., C. W. Park, Y. D. Kim y Y. Suh. 2001. Phylogenetic relationships in family Magnoliaceae inferred from $n d h F$ sequences. American Journal of Botany. 88:717-728.

Luna Vega, M. I. 2003. Magnolia dealbata. Taxones del bosque mesófilo de montaña de la Sierra Madre Oriental incluidos en la norma oficial mexicana. Herbario FCME, Departamento de Biología, Facultad de Ciencias, Universidad Nacional Autónoma de México. Bases de datos SNIB-CONABIO. Proyecto W025. México. D.F. 
Meyer, F. G. 1997. Magnolia. In Flora of North America North of Mexico, Flora of North America Editorial Committee (eds.). New York and Oxford. 3:3-10.

Ramírez-Bamonde, E. S., L. R. Sánchez-Velásquez y A. AndradeTorres. 2005. Seedling survival and growth of three species of mountain cloud forest in Mexico, under different canopy treatments. New Forests 30:95-101.

Sánchez-Velásquez, L. R. y M. del R. Pineda-López. 2006. Species diversity, structure and dynamics of two populations of an endangered species, Magnolia dealbata (Magnoliaceae). Revista de Biología Tropical. 54:997-1002.

Secretaría de Programación y Presupuesto. 1977. Carta de uso de suelo, G14C46, escala 1:50,000. México.

Secretaría de Programación y Presupuesto. 1978. Carta geológica, G14C46, escala 1:50,000. México.

Secretaría de Programación y Presupuesto. 1981. Carta estatal de climas, Nuevo León, escala 1:1, 000, 000. México.

Valdez-Tamez, V., R. Foroughbakhch-Pournavab y G. AlanísFlores. 2003. Distribución relictual del bosque mesófilo de montaña en el noreste de México. Ciencia, Universidad Autónoma de Nuevo León 6:360-365.

Vázquez, J. A. 1990. Taxonomy of the genus Magnolia (Magnoliaceae) in Mexico and Central America. M. Sc. thesis, University of Wisconsin, Madison. 68-78 p.

Vázquez, J. A. 1994. Magnolia (Magnoliaceae) in Mexico and Central America. Brittonia 1:1-23.

Vovides, A. 1988. Relación de plantas mexicanas raras o en peligro de extinción. Apéndice F. In Conservación en México: síntesis sobre vertebrados terrestres, vegetación y uso del suelo, O. Flores Villela y P. Gerez (eds.). Instituto Nacional de Investigaciones sobre Recursos Bióticos (INIREB), Xalapa, Veracruz. p. 289-302. 